\title{
¿UN IBERO ENTRE LOS EDUOS? NOTA A CIL XIII 2690
}

\author{
JAIME SILES \\ Universidad de Valencia \\ jaime.siles@uv.es
}

\section{RESUMEN}

El CIL XIII 2690 recoge una inscripción, encontrada en Autun y publicada en 1848 y recogida posteriormente en diferentes publicaciones, en la que hay un nombre que, aunque las lecturas difieren, podría ser el de un ibero, ATOBILES. Sin embargo, la información fotográfica más reciente inclina a leer NOBILIS.

PALABRAS CLAVE: epigrafía latina, onomástica ibérica.

\section{AN IBERIAN AMONG THE HAEDUI? ON CIL XIII 2690}

\section{ABSTRACT}

CIL XIII 2690 includes an inscription found in Autun and published in 1848 that bears a possible Iberian name, ATOBILES. However, recent photographs point to NOBILIS as being the most probable reading.

KEYWORDS: Latin epigraphy, Iberian personal names.

Agradezco mucho a los organizadores del XVIII Simposi d'Estudis Clàssics de la Secció Catalana de la SEEC el que se haya aceptado una comunicación tan humilde y breve como es la mía, que, por su carácter inseguro y casi conjetural, lleva un título entre interrogaciones. Como pueden ustedes ver en el resumen, el objeto de estudio es una inscripción recogida en el CIL XIII 2690, que, encastrada en la pared orientada a Este de la Maison des Caves-Joyaux, en Autun (fig. 1), fue encontrada en 1848 y publicada ese mismo año por A. Devoucoux y J. de Fontenay (1848: 201) en la revista Autun archéologique, por A. de Caumont (1862: 460) en Bulletin monumental en 1862, por É. Espérandieu (1910: no 1903) en su Recueil général des bas-reliefs, statues et bustes de la Gaule romaine; recogida luego por A. Rebourg (1993: 149, no 443, fig. 126) en su edición de la Carte archéologique de la Gaule 71/1 e incluida más tarde en Les villes antiques de la France (Frézouls 1997: 111) y, en fecha más reciente, por Yann Le Bohec (2015: 156, no 239, fig. 137 a y b) en Inscriptions de la cité des Éduens. Inscriptions sur pierre. Inscriptiones Latinae Galliae Lugudunensis (ILGL). 2. Aedui (L.Aed.), de donde tomo la información, ya que la que proporciona Otto Hirschfeld (1899: 421), editor del tomo XIII del CIL, no puede ser más escueta: se limita a decir que la ha buscado en vano (Ego frustra quaesivi).

Se trata de una estela de piedra arenisca en frontón triangular y con acroteras de una altura de $144 \mathrm{~cm}$ de alto y $59 \mathrm{~cm}$ de ancho. Las letras son irregulares y sus medidas varían entre los $6,5 \mathrm{~cm}$ de la tercera letra -la $O$ de 
$A T O-$, los 9 de la cuarta -la $B$ de BILES- y los 8 de la letra $D$, abreviatura de DIS, fórmula reducida de DIS MANIBVS. Esta mención a los dioses Manes inclina a pensar en una datación en torno al siglo II. La iconografía presenta una figura masculina de medio cuerpo que porta una pizarra de escritura en la mano izquierda y un objeto que, según Friederike Harl (2016), podría tratarse de un stilus, en la derecha. Hirschfeld (1899: 421), a partir de la información recibida y de grabados publicados por Caumont, dice que la figura representa a un varón (vir), que en su mano izquierda sostiene un cofre o arqueta (arculam) y, en la derecha, un anzuelo o garfio (uncinum), sin que esto último se pueda determinar muy bien, de modo que la iconografía no proporciona demasiados datos.

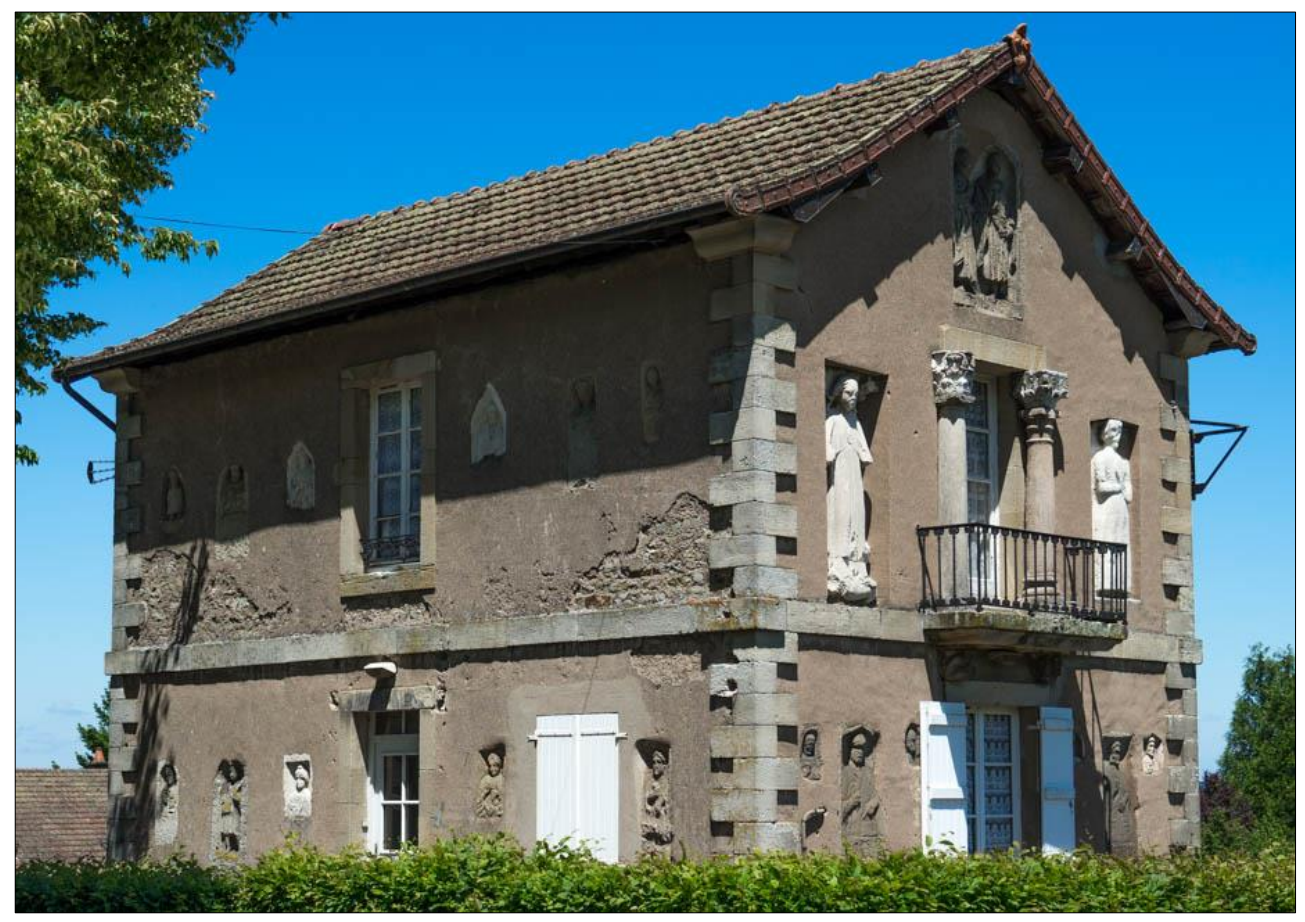

Fig. 1. Maison des Caves-Joyaux (Autun). (C Ubi Erat Lupa, Foto: Ortolf Harl.

Citada en el tomo I del Onomasticon provinciarum Europae Latinarum de Lőrincz (20052: 203), sin otra información que la que ya teníamos, se debe a Pierre-Yves Lambert el siguiente comentario, que Le Bohec (2015: 156) reproduce: "évoque [...] un nom ibère, Andebilis / Andobilis / Indebilis", lo que, a juicio de Le Bohec, merece cierto interés por haber aparecido en territorio de los eduos que, como es sabido, eran una tribu gala que, en época romana, ocupaba la actual Borgoña, siendo su capital Bibracte (Mont Beuvray), próxima a Autun, donde la estela se encontró.

La lectura e interpretación de esta inscripción funeraria pensamos que no es la propuesta por Le Bohec. Disponemos de una serie de fotografías, hechas en diciembre de 2015 por Ortolf Harl, en las que puede verse tanto la naturaleza del soporte como los detalles de las letras (fig. 2-3). La lectura ATOBILES propuesta por el CIL XIII 2690 y seguida por Le Bohec, que la mantiene y reproduce- ha sido modificada por Friederike Harl (2016), que lee NOBILIS. Esta 
disparidad de lecturas -sobre todo, en los primeros signos- obliga a una reconsideración de la suposición hecha por Lambert. Si se admite la lectura de Harl, tendríamos un nombre propio, procedente de un apelativo, lo que podría indicar que el difunto sería un liberto. En cambio, si se sigue la lectura de Caumont, del CIL y de Le Bohec, tendríamos un nombre aquitano o ibérico, cuyo primer elemento de la composición parecería galo. Todo depende de si en los primeros signos leemos $\mathrm{NO}$-como se observa en Harl-o si vemos en ellos $A T O$, que llevaría a comparar este nombre -ATOBILES - con el del caudillo ilergete, hermano de Mandonio, citado por Livio (22.21.2 y 25.34.6) como Indebilis o Indibilis; por Diodoro (26.22.11) como Indibeles; por Valerio Máximo (4.3.1), Apiano (Hisp. 37-38) y Zonaras (435) como Indibilis, y por Dion (16.57.42) como Indibolis, que María Lourdes Albertos Firmat (1966: 124-125), siguiendo a Antonio Tovar (1949: 163-166), considera variantes -junto con Andobales- "de un único nombre [...] cuya forma original parece ser Endobeles o quizá en versión en caracteres ibéricos, A.da.be.l.s", interpretable como "una formación híbrida, indoeuropea-ibera", en la que podría reconocerse el prefijo *ndhi- con valor superlativo y el vasco beltz 'negro' (Albertos 1966: 271 y 282). Gorrochategui (1984: 148) lo considera poco probable.

ATOBILES podría ser tanto un nombre personal ibérico como aquitano. ATO está abundantemente documentado en la Península Ibérica (Untermann 1965: 63, mapa 13), tanto en escritura ibérica (a.tu. atestiguado en Azaila, E.1.58; en Bílbilis, K.22.2; en Tarragona, C.18.6, y posiblemente en la forma a.tu.n.i.u. de Castellón, F.6.1; en Ensérune, B.1.349; en el plomo de Gandía, *G.20.1) ${ }^{1}$ como en escritura latina, en ésta con geminación consonántica, Atto (en Segovia: Atto, CIL II 2734; en Brunete, Madrid: Atto, CIL II 6338cc; en San Esteban de Gormaz: Atto, CIL II 2814; y en el nombre de un Caesaraugustanus en Palencia: Attus, CIL II 5764). En cuanto al segundo elemento, BILES, podría compararse con el ibérico beles ${ }^{2}$ que, en una inscripción de Cehegín (Caravaca, Murcia: CIL II 3537), escrita en caracteres latinos, presenta un nombre femenino Bileseton, en el que se puede reconocer la forma BILES, la misma que, según la lectura de Caumont, Hirschfeld y Le Bohec, aparece en la inscripción de Autun y que concuerda con la del primer elemento del nombre personal del ilergete Bilistages citado por Livio (34.11.2). Y podría compararse también con los aquitanos Belex, Belexconis, Belexeia, Belexennis, Belix, Harbelex, Harbelexsis, Harbelesteg... Otra posibilidad sería pensar que -BILES estaría encubriendo la forma ibérica BILOS, muy productiva en la formación de nombres propios compuestos, y que $A T O$ - fuera una forma reducida de los aquitanos ANDE- o ANDO- (Untermann 1965: 57, mapa 9) como Andere, Andereni, Anderes, Andereseni, Anderexo, Anderitia, Andos, Andossic, Andossius, Andossi, Andosso, Andossus, Andosten, Andostenni, Andostenno, Andoston, Andostonis, Andost, Andoxus, Andoxponni, Andus... (Gorrochategui 1984: 127-143).

\footnotetext{
1 Siles 1985: 45; Velaza 1991: 38; Silgo 1994: 31. Las inscripciones se citan según los MLH.

${ }^{2}$ Untermann 1965: 71, mapa 17; Siles 1985: 116-117; Untermann 1987: 299-300; Velaza 1991: 53-54.
} 

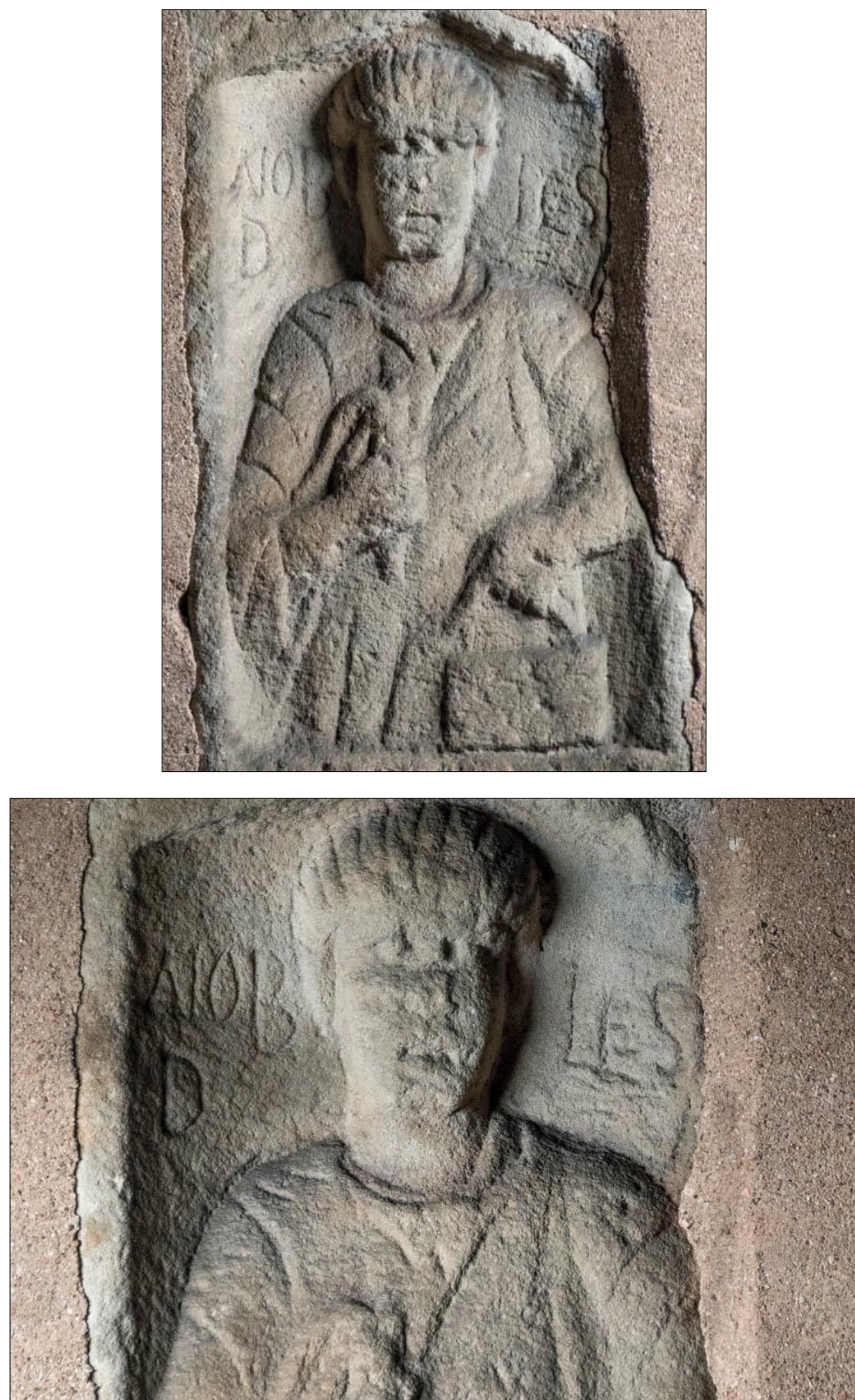

Fig. 2-3. Estela-retrato de Nobilis. (C) Ubi Erat Lupa, Fotos: Ortolf Harl.

ATOBILES podría ser, pues, tanto un nombre ibérico como uno aquitano: tanto en un caso como en otro podría pensarse que ATOBILES podría estar encubriendo fonéticamente un nombre personal */antobiles/ muy próximo en su forma al del nombre del caudillo ilergete transmitido por las fuentes clásicas como Andobales o Indibilis y cuya forma ibérica original parece ser Endobeles. Sin embargo, parece mucho más probable que la inscripción funeraria de Autun contenga no este nombre, sino NOBILIS, como se lee en la fotografía de Harl. Mientras no haya una lectura por completo segura y aceptada, no se puede afirmar que el nombre que aparece escrito en la estela funeraria de Autun sea el de un aquitano o un ibero, 
por más que lo parezca. La cautela debida obliga a ello: es decir, a mantener entre signos de interrogación el título del trabajo aquí presentado, toda vez que, a juzgar por las fotografías, parece que hay que aceptar la lectura que se deduce de ellas: NOBILIS y no ATOBILES.

\section{BIBLIOGRAFÍA}

AlBeRTOS FIRMAT, M. L. (1966), La onomástica personal primitiva de Hispania (Tarraconense y Bética), Salamanca, Universidad de Salamanca.

CAUMONT, A. de (1862), “Nécrologie gallo-romaine ou excursions dans les musées lapidaires de France", Bulletin monumental, 28, 433-460.

Devoucoux, A. y Fontenay, J. de (1848), "Symbole de l'arca", Autun archéologique, s/n, 201-203.

ESPÉRANDIEU, É. (1910), Recueil général des bas-reliefs, statues et bustes de la Gaule romaine, vol. III: Lyonnaise - Première partie, París, Imprimerie nationale.

FrÉzOUlS, E. (ed.) (1997), Les villes antiques de la France, vol. III: Lyonnaise 1. Autun Chartres - Nevers, París, De Boccard.

Gorrochategui ChurruCA, J. (1984), Onomástica indígena de Aquitania, Bilbao, EHU.

HARL, F. y O. (2016), “Porträtstele des Nobilis", Ubi Erat Lupa [en línea]. Disponible en: $<$ lupa.at/25779> [consulta: 3 octubre 2018].

HIRSCHFELD, O. (1899), Corpus Inscriptionum Latinarum XIII. Inscriptiones trium Galliarum et Germaniae Latinae I.1. Inscriptiones Aquitaniae et Lugudunensis, Berlín, Reimer.

LE BOHEC, Y. (2015), Inscriptions de la cité des Éduens. Inscriptions sur pierre. Inscriptiones Latinae Galliae Lugudunensis (ILGL). 2. Aedui (L.Aed.), Barcelona, Universitat de Barcelona.

LŐRINCZ, B. et al. (2005² [1994]), Onomasticon provinciarum Europae Latinarum, vol. I: Aba - Bysanus, Budapest, Opitz.

MLH = UNTERMANN, J. (1975-1997), Monumenta linguarum Hispanicarum, 4 vols., Wiesbaden, Reichert.

RebourG, A. (1993), Carte archéologique de la Gaule, vol. 71/1: Autun, París, Maison des Sciences de l'Homme.

SILES, J. (1985), Léxico de inscripciones ibéricas, Madrid, Ministerio de Cultura.

SILGO GAUCHE, L. (1994), Léxico ibérico, Valencia, Real Academia de Cultura Valenciana.

TOVAR, A. (1949), Estudios sobre las primitivas lenguas hispánicas, Buenos Aires, Universidad de Buenos Aires.

UNTERMANN, J. (1965), Elementos de un atlas antroponímico de la Hispania antigua, Madrid, CSIC / Universidad de Madrid.

UNTERMANN, J. (1987), "Repertorio antroponímico ibérico", Archivo de Prehistoria Levantina, 17, 289-318.

VelazA, J. (1991), Léxico de inscripciones ibéricas (1976-1989), Barcelona, Universitat de Barcelona. 